\title{
Double-Zipper: Multiple Access with ZigZag Decoding
}

\author{
Mohammad Kazemi \\ EEE Dept., Bilkent University \\ Ankara, Turkey \\ kazemi@ee.bilkent.edu.tr
}

\author{
Tolga M. Duman \\ EEE Dept., Bilkent University \\ Ankara, Turkey \\ duman@ee.bilkent.edu.tr
}

\author{
Muriel Médard \\ Dept. of EECS, MIT \\ Cambridge, MA \\ medard@mit.edu
}

\begin{abstract}
As a building block toward a simple and scalable solution to massive random access, we consider two-user multiple access with ZigZag decoding, with no need for any coordination or codebook differentiation. We derive closed-form bounds on the achievable sum-rates of the original ZigZag and a modified version of it, called double-zipper ZigZag, for both cases of perfect and imperfect channel state information (CSI). We also show that performances of both versions of ZigZag approach that of optimal coordinated time-sharing in the high signal to noise ratio regime, even in the presence of CSI errors.
\end{abstract}

Index Terms-Random access, ZigZag decoding, capacity bounds, imperfect CSI.

\section{INTRODUCTION}

Two parallel approaches have been developed in the previous literature to address the problem of multiple access communications. The first approach follows the footsteps of Ahlswede [1] and Liao [2], and considers multiple access channels (MACs) for which codebooks of individual users are distinct and the channels are perfectly synchronized. The receiver, having access to the users codebooks, can decode the individual messages using different techniques. The rate region achievable via this approach is named as the CoverWyner rate region [3]. Considering Gaussian noise channels, at high signal to noise ratios (SNRs), the rate region approaches the time-sharing region, while at low SNRs, it becomes a rectangular one [4], [5]. While it is possible to achieve the MAC capacity via this approach, it does not scale beyond a few users. In particular, it is impossible to assign distinct codebooks to a massive number of users and keep track of the transmitting ones at the receiver when they are only sporadically active. Furthermore, it is not practical to have all the users transmissions perfectly synchronized in time.

The second approach which has originated from Univ. of Hawaii in early 1970s is the simple ALOHA protocol [6] and its variants, which traditionally ignore the channel coding problem. This approach is based on collision avoidance, i.e., colliding packets are discarded at the receiver. While this approach is scalable in terms of the number of users, its overall throughput performance is highly inferior. More recent literature considers some extensions of ALOHA along with successive interference cancellation [7] to improve its throughput; however, this approach does not solve the problem of massive uncoordinated random access with near information theoretic limits either.
A recent information theoretic formulation for the massive random access problem is developed by Polyanskiy [9], assuming that the users utilize the same codebook. He defines a $K_{a}$-user MAC code as a collection of norm-constrained vectors, where the noisy sum of any $K_{a}$ of them can be decoded with a certain probability of error. $\mathrm{He}$ also presents some bounds on the capacity of the massive random access systems. However, in this formulation too, the users' transmissions are assumed fully synchronized, and it appears difficult to achieve these information theoretic limits in practice [10], [11].

In this paper, with the aim of facilitating massive random access in a practical manner, we consider the use of ZigZag decoding [8]. The basic idea is to transmit the same packet twice from both users in such a way that they experience different delays at the receiver, which could be due to the differences in channel delays or may be introduced artificially by proper randomization at the transmitters. Then, the receiver performs interference cancellation based on the received signals only, i.e., without the need for decoding of users' messages. Once the interference is cancelled, each user's message is decoded separately. Namely, the users can adopt the same codebooks (designed for single user communications), i.e., this scheme can be used as a building block for massive random access.

Some aspects of ZigZag decoding have been studied in the previous literature. In [12], the authors compare the throughput performances of ALOHA and carrier-sense multiple access (CSMA) schemes with ZigZag decoding. An algebraic representation of collisions which views each collision as a linear combination of the original packets is presented in [13]. This scheme outperforms not only the ALOHA-type schemes from a delay perspective, but also the centralized scheduling solutions. In [14], two ACK policies are proposed that stabilize the random access system with ZigZag decoding. In [15], the authors show that ZigZag decoding can be seen as an instance of belief propagation in the high SNR regime. Building on this observation, they present a simple soft-decoding version, called SigSag. In [16], to deal with the error floor problem in a frameless structure, a scheme with two-bit feedback called enhanced ZigZag decodable frameless ALOHA (E-ZDFA) is proposed. In [17], ZigZag decodable (ZD) codes are proposed for distributed storage systems. A fountain coding system based on ZD codes is proposed in [18].

In this paper, we obtain lower and upper bounds on the 
achievable sum-rate of a two-user system with ZigZag decoding in a non-unit gain channel with imperfect CSI. We also show that the gap between the lower bound on the achievable sum-rate of ZigZag decoding and the upper bound on the timesharing sum-rate becomes a constant at high SNRs. That is, the performance of ZigZag decoding approaches that of optimal coordinated time-sharing based solutions asymptotically. Note that the ZigZag scheme is applicable even when the users utilize the same codebooks. Hence, once it is extended to handle more than two users, it would offer a solution to the problem of massive random access with suitable transmission protocols [9] [19].

The rest of the paper is organized as follows. In Section II, the system model is introduced, and a short explanation of ZigZag decoding process is given along with a characterization of the resulting noise. In Section III, we propose a variation of ZigZag decoding, which we call double-zipper ZigZag. Bounds on the achievable sum-rate of the ZigZag decoding for both cases of perfect and imperfect CSI are also obtained. Numerical examples are provided in Section IV, and finally, the paper is concluded in Section V.

\section{SySTEM MODEL}

We have two users, each interested in transmitting a packet of length $N$ to a receiver. Since there is no coordination between the users or with the receiver, collisions occur. To resolve the collisions, we rely on differences in the delays experienced by the users' transmissions. To be more precise, we have two time intervals. In each time interval, the users simultaneously send their packets without synchronization, hence the packets experience different delays. Note that while the delays may be naturally occurring, they may also be introduced by randomizing the packet transmission times at the users. The receiver then employs ZigZag decoding to decouple the users' packets using its observations in the two time intervals.

Let the total transmission duration, and the first and the second delay differences be $T=(2+\alpha) N, L_{1}=\beta_{1} N$ and $L_{2}=\beta_{2} N$, respectively, with $\alpha \geq 0$ (obviously $\alpha \geq \beta_{1}+$ $\beta_{2}$, but independent of delay differences), and without loss of generality, $\beta_{2}>\beta_{1} \geq 0$.

Assuming that the users transmit independently with equal power $P$, we now present some capacity expressions, which are used for comparisons later on. For the sum-rate capacity (per channel use), we have

$$
C_{S C}=\log \left(1+\rho\left(\left|h_{1}\right|^{2}+\left|h_{2}\right|^{2}\right)\right)
$$

where $\rho \triangleq \frac{P}{\sigma^{2}}$ is SNR and $h_{k}$ is the channel gain of the $k$-th user. While for the time-sharing sum-rate capacity (per channel use), we have

$$
C_{T S}=\frac{1}{2} \log \left(1+2 \rho\left|h_{1}\right|^{2}\right)+\frac{1}{2} \log \left(1+2 \rho\left|h_{2}\right|^{2}\right) .
$$

We will also use as reference the expression derived in [21] using a deterministic approach, which is within 0.5 bits per user of the capacity,

$$
C_{A D T}=\left[\log \left(\rho \max \left\{\left|h_{1}\right|^{2},\left|h_{2}\right|^{2}\right\}\right)\right]^{+},
$$

where $\lceil a\rceil^{+}$denotes the least non-negative integer greater than or equal to $a$.

\section{Characterization OF ZigZag Decoding}

\section{A. Double-Zipper ZigZag}

In the original ZigZag decoding process [13], the decoding starts from one side (e.g., left to right) through the received overlapped packets. In order to accumulate less noise, we propose a modified version of ZigZag decoding, called doublezipper ZigZag, in which the decoding is performed from both sides through the middle of the overlapped packets. Double-zipper ZigZag process proceeds as follows (assuming $L_{2}>L_{1}$ ): In the first decoding step, using the received signals in the second time interval, the first $L_{2}$ symbols of the first user and the last $L_{2}$ symbols of the second user are observed without interference. Next, $L_{2}-L_{1}$ symbols of each user are removed of interference in each step using the observations of the previous step. The process proceeds in the same manner until both packets are interference free.

Let us describe the process mathematically. First, consider unit gain channels with perfect CSI at the receiver. As examples, decoding processes of the original and double-zipper ZigZag are depicted in Fig. 1 and Fig. 2, respectively, with $N=5, L_{1}=1$ and $L_{2}=3$, where $n_{i, j}$ is the additive white Gaussian noise (AWGN) of the $j$-th time slot of the $i$-th time interval at the receiver with variance $\sigma_{n}^{2}$, and $s_{i, j}$ is the $j$-th symbol of the $i$-th user. Based on Fig. 2, in the first step of the decoding process, three interference free symbols of each user are

$$
\begin{aligned}
& \hat{s}_{1,1}=y_{2,1}=s_{1,1}+n_{2,1}, \\
& \hat{s}_{1,2}=y_{2,2}=s_{1,2}+n_{2,2}, \\
& \hat{s}_{1,3}=y_{2,3}=s_{1,3}+n_{2,3}, \\
& \hat{s}_{2,3}=y_{2,6}=s_{2,3}+n_{2,6}, \\
& \hat{s}_{2,4}=y_{2,7}=s_{2,4}+n_{2,7}, \\
& \hat{s}_{2,5}=y_{2,8}=s_{2,5}+n_{2,8},
\end{aligned}
$$

where $\hat{s}_{i, j}$ is the interference free $j$-th symbol of the $i$-th user, and $y_{i, j}$ is the $j$-th received signal in the $i$-th time interval. Also, using (4), the remaining four symbols are freed of interference in the second step, resulting in

$$
\begin{aligned}
& \hat{s}_{2,1}=y_{1,2}-\hat{s}_{1,2}=s_{2,1}+n_{1,2}-n_{2,2}, \\
& \hat{s}_{2,2}=y_{1,3}-\hat{s}_{1,3}=s_{2,2}+n_{1,3}-n_{2,3}, \\
& \hat{s}_{1,4}=y_{1,4}-\hat{s}_{2,3}=s_{1,4}+n_{1,4}-n_{2,6}, \\
& \hat{s}_{1,5}=y_{1,5}-\hat{s}_{2,4}=s_{1,5}+n_{1,5}-n_{2,7} .
\end{aligned}
$$

Employing the double-zipper ZigZag strategy, the equivalent system model can be written as

$$
\hat{\mathbf{s}}=\mathbf{s}+\mathbf{A n},
$$

$$
\begin{aligned}
& \text { where } \hat{\mathbf{s}} \quad=\quad\left[\hat{s}_{1,1}, \ldots, \hat{s}_{1, N}, \hat{s}_{2,1}, \ldots, \hat{s}_{2, N}\right]^{\mathrm{T}} \\
& \text { is the } 2 N \times 1 \text { vector of decoded symbols, }
\end{aligned}
$$




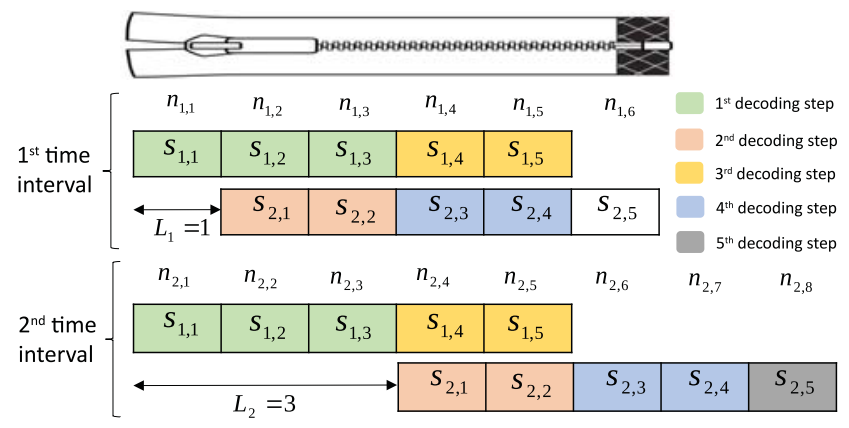

Fig. 1: Original ZigZag process for $N=5, L_{1}=1$ and $L_{2}=3$.

$\mathbf{n}=\left[n_{1,1}, \ldots, n_{1, N+L_{1}}, n_{2,1}, \ldots, n_{2, N+L_{2}}\right]^{\mathrm{T}}$ is the $\left(2 N+L_{1}+L_{2}\right) \times 1$ vector of received AWGN noise terms, $\mathbf{s}=\left[s_{1,1}, \ldots, s_{1, N}, s_{2,1}, \ldots, s_{2, N}\right]^{\mathrm{T}}$ is the $2 N \times 1$ vector of transmitted symbols, and $\mathbf{A}$ is the $2 N \times\left(2 N+L_{1}+L_{2}\right)$ coefficient matrix representing the aggregate noise terms due to the ZigZag decoding process. Note that the same model holds for the original ZigZag, albeit with a different matrix of noise coefficients.

\section{B. Noise Covariance Matrix}

Let $\mathbf{n}$ be a zero-mean complex circularly symmetric Gaussian vector with independent elements of variance $\sigma_{n}^{2}$. Then the noise covariance matrix becomes $\mathbf{R}_{n}=\sigma_{n}^{2} \mathbf{A} \mathbf{A}^{\mathrm{T}}$.

We first review the original ZigZag decoding process. In its first step, $L_{2}$ symbols are available free of interference with only one input noise term. Next, in each middle step, $\left(L_{2}-L_{1}\right)$ symbols are obtained (with the exception of $r \triangleq\left(N-L_{2}\right) \bmod \left(L_{2}-L_{1}\right)$ symbols in third-to-last and second-to-last steps), accumulating an additional input noise term in each step. Finally, $L_{1}$ symbols are obtained in the last step with one input noise term. The process is completed after $\left\lceil\frac{N-L_{2}}{L_{2}-L_{1}}\right]+\left\lceil\frac{N-L_{1}}{L_{2}-L_{1}}\right\rceil+2=2\left\lceil\frac{N-L_{2}}{L_{2}-L_{1}}\right\rceil+3$ steps.

On the other hand, the double-zipper ZigZag decoding process has $\left[\frac{N-L_{2}}{L_{2}-L_{1}}\right]+1$ steps, which translates to almost half of that of the original ZigZag for large $N$, leading to less noise accumulation. To be exact, $2 L_{2}$ symbols are available in its first step with one input noise term. Next, $2\left(L_{2}-L_{1}\right)$ symbols are obtained in each step $(2 r$ symbols in the last step), accumulating an additional input noise term in each step. Summing up, we can say that:

Lemma 1. In the original ZigZag decoding process, the diagonal of the noise covariance matrix $\mathbf{R}_{n}$ has $L_{1}+L_{2}$ elements equal to $\sigma_{n}^{2}, 2 M+1$ groups of $L_{2}-L_{1}$ elements each with value $k \sigma_{n}^{2}, k=2, \ldots, 2 M+2, r$ elements equal to $(2 M+3) \sigma_{n}^{2}$, and $r$ elements equal to $(2 M+4) \sigma_{n}^{2}$, where $M \triangleq\left\lfloor\frac{N-L_{2}}{L_{2}-L_{1}}\right\rfloor$.

In the double-zipper ZigZag decoding process, the diagonal of the noise covariance matrix $\mathbf{R}_{n}$ has $2 L_{2}$ elements equal to $\sigma_{n}^{2}, M$ groups of $2\left(L_{2}-L_{1}\right)$ elements each with value $k \sigma_{n}^{2}$, $k=2, \ldots, M+1$, and $2 r$ elements equal to $(M+2) \sigma_{n}^{2}$.

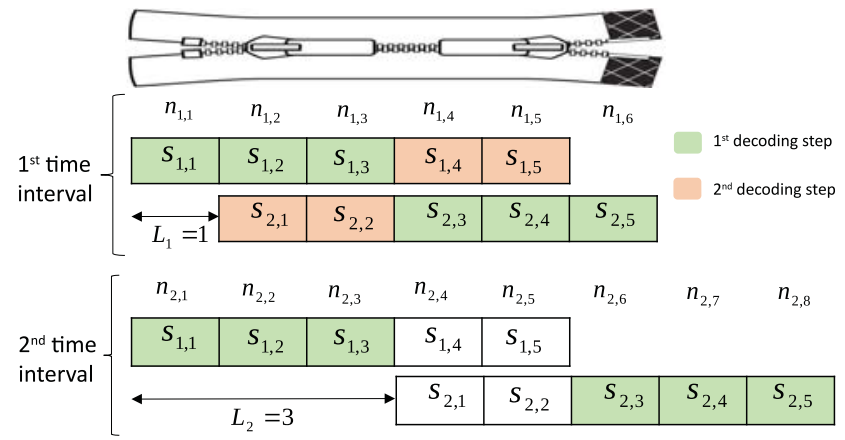

Fig. 2: Double-zipper ZigZag process for $N=5, L_{1}=1$ and $L_{2}=3$.

\section{Bounds on the Sum-Rate With Perfect CSI}

\section{A. Unit Channel Gains}

Using the equivalent model of (6), the ZigZag achievable sum-rate (per channel use) becomes

$$
C_{Z Z}=\frac{1}{2 N} \log \left(\frac{\operatorname{det}\left(\mathbf{R}_{n}+P \mathbf{I}\right)}{\operatorname{det}\left(\mathbf{R}_{n}\right)}\right),
$$

where $\mathbf{I}$ is the identity matrix.

The next lemma proves that if we replace the correlated noise terms with independent terms of the same variances, the mutual information will not increase if the codebook elements are independent.

Lemma 2. Let $Y^{N}=X^{N}+Z^{N}$ with $X^{N}=$ $\left[X_{1}, X_{2}, \ldots, X_{N}\right]$ and $Z^{N}=\left[Z_{1}, Z_{2}, \ldots, Z_{N}\right]$. Assume that $X_{i}$ 's, and the vectors $X^{N}$ and $Z^{N}$ are independent, however, $Z_{i}$ 's are correlated. Then, $I\left(X^{N} ; Y^{N}\right) \geq \sum_{i=1}^{N} I\left(X_{i} ; Y_{i}\right)$.

Proof: We first expand the mutual information term $I\left(X^{N} ; Y^{N}\right)$ as

$$
\begin{aligned}
I\left(X^{N} ; Y^{N}\right) & =\sum_{i=1}^{N} I\left(X^{N} ; Y_{i} \mid Y^{i-1}\right) \\
& =\sum_{j=1}^{N} \sum_{i=1}^{N} I\left(X_{j} ; Y_{i} \mid X^{j-1}, Y^{i-1}\right) .
\end{aligned}
$$

Since mutual information is non-negative, keeping only the terms with $j=i$, we have

$$
I\left(X^{N} ; Y^{N}\right) \geq \sum_{i=1}^{N} I\left(X_{i} ; Y_{i} \mid X^{i-1}, Y^{i-1}\right) .
$$

Since $X_{i}$ is independent of $X^{i-1}$ and $Y^{i-1}$ (from the convexity of mutual information $I(U ; V)$ in $p(v \mid u)$ for fixed $p(u)$ [see [20], p. 25]), $I\left(X_{i} ; Y_{i} \mid X^{i-1}, Y^{i-1}\right) \geq I\left(X_{i} ; Y_{i}\right)$, and the claim follows.

Theorem 1. The achievable sum-rates per channel use of the double-zipper and the original ZigZag decoding algorithms can be lower bounded as

$$
\begin{gathered}
C_{D Z} \geq \beta_{2} \log (1+\rho)+\frac{r}{N} \log \left(1+\frac{\rho}{M+2}\right) \\
+\left(\beta_{2}-\beta_{1}\right) \sum_{k=1}^{M} \log \left(1+\frac{\rho}{k+1}\right)
\end{gathered}
$$


and

$$
\begin{aligned}
C_{O Z} \geq & \frac{\beta_{1}+\beta_{2}}{2} \log (1+\rho)+\frac{\beta_{2}-\beta_{1}}{2} \sum_{k=1}^{1+2 M} \log \left(1+\frac{\rho}{k+1}\right) \\
& +\frac{r}{2 N}\left(\log \left(1+\frac{\rho}{2 M+3}\right)+\log \left(1+\frac{\rho}{2 M+4}\right)\right),
\end{aligned}
$$

respectively.

Proof: Using Lemma 2, we have

$$
C_{Z Z} \geq \frac{1}{2 N} \log \left(\frac{\operatorname{det}\left(\mathbf{R}_{n}^{\prime}+P \mathbf{I}_{2 N}\right)}{\operatorname{det}\left(\mathbf{R}_{n}^{\prime}\right)}\right),
$$

where $\mathbf{R}_{n}^{\prime}$ is a diagonal matrix with the same diagonal elements as $\mathbf{R}_{n}$. Next, using Lemma 1, we have

$$
\begin{aligned}
\operatorname{det}\left(\mathbf{R}_{n}^{\prime}\right) & =\underbrace{\left(\sigma_{n}^{2}\right)^{2 L_{2}}}_{\text {first step }} \underbrace{\left((M+2) \sigma_{n}^{2}\right)^{2 r}}_{\text {last step }} \underbrace{\prod_{k=1}^{M}\left((k+1) \sigma_{n}^{2}\right)^{2\left(L_{2}-L_{1}\right)}}_{\text {middle steps }} \\
& =\left(\sigma_{n}^{2}\right)^{2 N}(M+2)^{2 r} \prod_{k=1}^{M}(k+1)^{2\left(L_{2}-L_{1}\right)} .
\end{aligned}
$$

Also, we have

$$
\begin{aligned}
& \operatorname{det}\left(\mathbf{R}_{n}^{\prime}+P \mathbf{I}\right) \\
& =\underbrace{\left(\sigma_{n}^{2}+P\right)^{2 L_{2}}}_{\text {first step }} \underbrace{\left((M+2) \sigma_{n}^{2}+P\right)^{2 r}}_{\text {last step }} \underbrace{\prod_{k=1}^{M}\left((k+1) \sigma_{n}^{2}+P\right)^{2\left(L_{2}-L_{1}\right)}}_{\text {middle steps }} \\
& =\left(\sigma_{n}^{2}\right)^{2 N}(1+\rho)^{2 L_{2}}((M+2)+\rho)^{2 r} \prod_{k=1}^{M}((k+1)+\rho)^{2\left(L_{2}-L_{1}\right)} .
\end{aligned}
$$

Substituting (13) and (14) into (12), gives (10), the lower bound on the achievable sum-rate of the double-zipper ZigZag process. In a similar manner, the lower bound on the achievable sum-rate of the original ZigZag process is obtained as in (11).

\section{B. Non-Unit Channel Gains}

We now consider non-unit channel gains, which are taken as constant during the two time intervals. We first assume that the receiver knows all the channel gains perfectly.

In a similar manner to the previous section, we can obtain an equivalent model of the ZigZag decoding for the non-unit gain channels as follows

$$
\hat{\mathbf{y}}=\mathbf{H s}+\mathbf{A n},
$$

where $\hat{\mathbf{y}}=\mathbf{H} \hat{\mathbf{s}}$, and $\mathbf{H}$ is a diagonal matrix with $\mathbf{H}_{k, k}=h_{1}$ for $k=1, \ldots, N$ and $\mathbf{H}_{k, k}=h_{2}$ for $k=N+1, \ldots, 2 N$, where $h_{k}$ is the channel gain of the $k$-th user. Using this equivalent model, the achievable sum-rate (per channel use) for channels with non-unit gains becomes

$$
C_{Z Z}=\frac{1}{2 N} \log \left(\frac{\operatorname{det}\left(\mathbf{R}_{n}+P \mathbf{H H}^{\mathrm{H}}\right)}{\operatorname{det}\left(\mathbf{R}_{n}\right)}\right) .
$$

In the double-zipper ZigZag decoding, half of the symbols (to be decoded in each step) are decoded using the first channel gain and the other half using the second one. Using this fact, similar to Theorem 1, we obtain a lower bound on the achievable sum-rate (per channel use) of the double-zipper ZigZag for channels with non-unit gains as follows

$$
\begin{aligned}
C_{D Z} \geq & \frac{\beta_{2}}{2}\left(\log \left(1+\rho\left|\bar{h}_{1}\right|^{2}\right)+\log \left(1+\rho\left|\bar{h}_{2}\right|^{2}\right)\right) \\
& +\frac{\beta_{2}-\beta_{1}}{2} \sum_{k=1}^{M}\left(\log \left(1+\frac{\rho\left|\bar{h}_{1}\right|^{2}}{k+1}\right)+\log \left(1+\frac{\rho\left|\bar{h}_{2}\right|^{2}}{k+1}\right)\right) \\
& +\frac{r}{2 N}\left(\log \left(1+\frac{\rho\left|\bar{h}_{1}\right|^{2}}{M+2}\right)+\log \left(1+\frac{\rho\left|\bar{h}_{2}\right|^{2}}{M+2}\right)\right) .
\end{aligned}
$$

On the other hand, in the original ZigZag process, the associated channel gain changes in each step, and we obtain

$$
\begin{aligned}
C_{O Z} \geq & \frac{\beta_{1}}{2}\left(\log \left(1+\rho\left|\bar{h}_{1}\right|^{2}\right)+\log \left(1+\rho\left|\bar{h}_{2}\right|^{2}\right)\right) \\
& +\frac{\beta_{2}-\beta_{1}}{2} \sum_{k=0}^{M}\left(\log \left(1+\frac{\rho\left|\bar{h}_{1}\right|^{2}}{2 k+1}\right)+\log \left(1+\frac{\rho\left|\bar{h}_{2}\right|^{2}}{2 k+2}\right)\right) \\
& +\frac{r}{2 N}\left(\log \left(1+\frac{\rho\left|\bar{h}_{1}\right|^{2}}{2 M+3}\right)+\log \left(1+\frac{\rho\left|\bar{h}_{2}\right|^{2}}{2 M+4}\right)\right) .
\end{aligned}
$$

\section{ZigZag DeCoding With IMPERfect CSI}

\section{A. Bounds on the Sum-Rate With Imperfect CSI}

We now assume that only estimates of channel gains are available to the receiver, $h_{k}=\bar{h}_{k}+\tilde{h}_{k}$, where $\bar{h}_{k}$ and $\tilde{h}_{k}$ are the estimated channel gain and the channel estimation error of the $k$-th user, respectively. The channel estimation error, $\tilde{h}_{k}$, is modeled as a zero-mean random variable with known variance $\sigma_{h}^{2}$, which is inversely proportional to the SNR, i.e., $\sigma_{h}^{2}=\frac{c}{\rho}$ for some constant $c$.

Using the results in [22], lower and upper bounds on the time-sharing sum-rate capacity (per channel use) with imperfect CSI can be obtained as

$$
C_{T S} \geq \frac{1}{2} \log \left(1+\frac{2 \rho\left|\bar{h}_{1}\right|^{2}}{2 c+1}\right)+\frac{1}{2} \log \left(1+\frac{2 \rho\left|\bar{h}_{2}\right|^{2}}{2 c+1}\right)
$$

and

$$
C_{T S} \leq \frac{1}{2} \log \left(1+2 c+2 \rho\left|\bar{h}_{1}\right|^{2}\right)+\frac{1}{2} \log \left(1+2 c+2 \rho\left|\bar{h}_{2}\right|^{2}\right) .
$$

For the ZigZag decoding with imperfect CSI, we obtain the same equivalent model as (15) with $\hat{\mathbf{y}}=\overline{\mathbf{H}} \hat{\mathbf{s}}$, where $\overline{\mathbf{H}}$ is a diagonal matrix with $\overline{\mathbf{H}}_{k, k}=\bar{h}_{1}$ for $k=1, \ldots, N$ and $\overline{\mathbf{H}}_{k, k}=\bar{h}_{2}$ for $k=N+1, \ldots, 2 N$. Again from [22], we get

$$
C_{Z Z} \geq \log \left(\left|\rho\left(c \mathbf{I}+\mathbf{A} \mathbf{A}^{\mathrm{T}}\right)^{-1} \overline{\mathbf{H}} \overline{\mathbf{H}}^{\mathrm{H}}+\mathbf{I}\right|\right)
$$

and

$$
C_{Z Z} \leq \log \left(\left|\left(\mathbf{A A}^{\mathrm{T}}\right)^{-1}\left(c \mathbf{I}+\rho \overline{\mathbf{H}} \overline{\mathbf{H}}^{\mathrm{H}}\right)+\mathbf{I}\right|\right) .
$$




\section{B. Sum-Rate Gap With Imperfect CSI}

Keeping only the diagonals of the noise covariance matrix and using the results in [22], we can obtain further lower bounds on the achievable sum-rate of the double-zipper and the original ZigZag with imperfect CSI as

$$
\begin{aligned}
C_{D Z} \geq & \frac{\beta_{2}}{2}\left(\log \left(1+\frac{\rho\left|\bar{h}_{1}\right|^{2}}{c+1}\right)+\log \left(1+\frac{\rho\left|\bar{h}_{2}\right|^{2}}{c+1}\right)\right) \\
& +\frac{\beta_{2}-\beta_{1}}{2} \sum_{k=1}^{M}\left(\log \left(1+\frac{\rho\left|\bar{h}_{1}\right|^{2}}{k+c+1}\right)+\log \left(1+\frac{\rho\left|\bar{h}_{2}\right|^{2}}{k+c+1}\right)\right) \\
& +\frac{r}{2 N}\left(\log \left(1+\frac{\rho\left|\bar{h}_{1}\right|^{2}}{M+c+2}\right)+\log \left(1+\frac{\rho\left|\bar{h}_{2}\right|^{2}}{M+c+2}\right)\right)
\end{aligned}
$$

and

$$
\begin{aligned}
C_{O Z} \geq & \frac{\beta_{1}}{2}\left(\log \left(1+\frac{\rho\left|\bar{h}_{1}\right|^{2}}{c+1}\right)+\log \left(1+\frac{\rho\left|\bar{h}_{2}\right|^{2}}{c+1}\right)\right) \\
& +\frac{\beta_{2}-\beta_{1}}{2} \sum_{k=0}^{M}\left(\log \left(1+\frac{\rho\left|\bar{h}_{1}\right|^{2}}{2 k+c+1}\right)+\log \left(1+\frac{\rho\left|\bar{h}_{2}\right|^{2}}{2 k+c+2}\right)\right) \\
& +\frac{r}{2 N}\left(\log \left(1+\frac{\rho\left|\bar{h}_{1}\right|^{2}}{2 M+c+3}\right)+\log \left(1+\frac{\rho\left|\bar{h}_{2}\right|^{2}}{2 M+c+4}\right)\right),
\end{aligned}
$$

respectively, which are extensions of lower bounds in Section III.A to the case of imperfect CSI. In the high SNR regime, (23) can be simplified to

$$
\begin{aligned}
C_{M Z} \geq & \log (\rho)+\log \left(\left|\bar{h}_{1}\right|\left|\bar{h}_{2}\right|\right)-\beta_{2} \log (c+1) \\
& -\left(\beta_{2}-\beta_{1}\right) \log \left(\frac{\Gamma(M+c+2)}{\Gamma(c+2)}\right)-\frac{r}{N} \log (M+c+2),
\end{aligned}
$$

using $\beta_{2}+\frac{r}{N}+M\left(\beta_{2}+\beta_{1}\right)=1$ and $\prod_{k=a}^{b}(k+c)=\frac{\Gamma(b+c+1)}{\Gamma(a+c)}$.

Also, in the high SNR regime, the upper bound on the timesharing sum-rate capacity (20) can be simplified to

$$
C_{T S} \leq \log (\rho)+1+\log \left(\left|\bar{h}_{1}\right|\left|\bar{h}_{2}\right|\right) .
$$

Using (25) and (26), the gap between the upper bound on the time-sharing sum-rate capacity and the lower bound on the achievable sum-rate of the double-zipper ZigZag in high SNRs becomes

$$
\begin{aligned}
\lim _{\rho \rightarrow \infty} \Delta C_{D Z}= & 1+\beta_{2} \log (c+1)+\frac{r}{N} \log (M+c+2) \\
& +\left(\beta_{2}-\beta_{1}\right) \log \left(\frac{\Gamma(M+c+2)}{\Gamma(c+2)}\right) .
\end{aligned}
$$

In the same manner, the gap between the upper bound on the time-sharing sum-rate capacity and the lower bound on the achievable sum-rate of the original ZigZag in high SNRs can be obtained as

$$
\begin{aligned}
\lim _{\rho \rightarrow \infty} \Delta C_{O Z}= & +\beta_{1} \log (c+1)+(M+1)\left(\beta_{2}-\beta_{1}\right) \\
& +\frac{\beta_{2}-\beta_{1}}{2} \log \left(\frac{\Gamma\left(M+\frac{3+c}{2}\right) \Gamma\left(M+\frac{c}{2}+2\right)}{\Gamma\left(\frac{c+1}{2}\right) \Gamma\left(\frac{c}{2}+1\right)}\right) \\
& +\frac{r}{2 N} \log ((2 M+c+3)(2 M+c+4)) .
\end{aligned}
$$

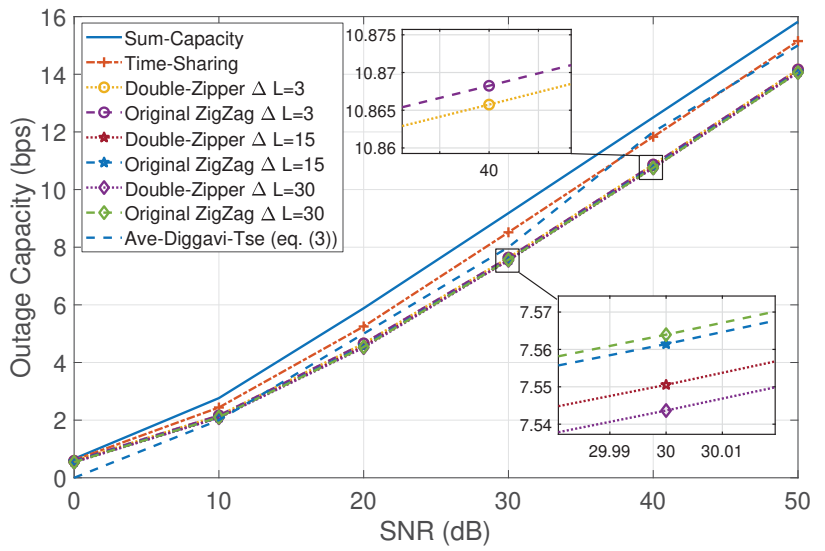

Fig. 3: Outage capacity with perfect CSI.

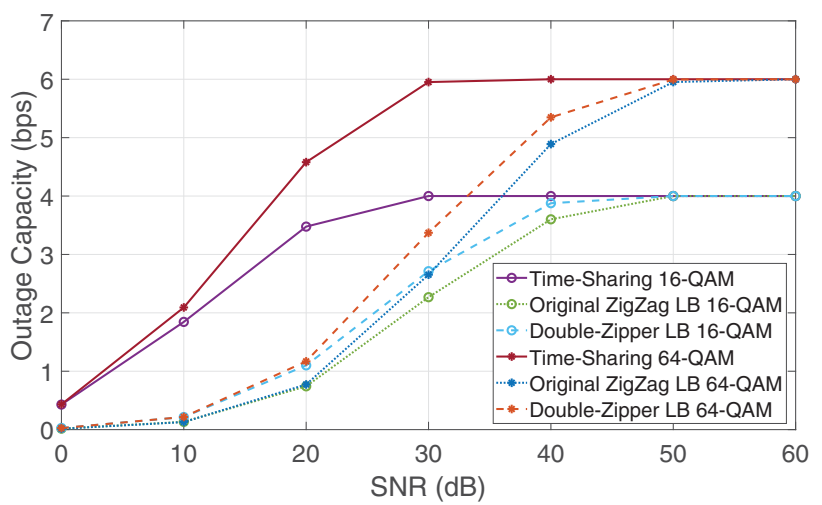

Fig. 4: Finite-alphabet achievable rate with perfect CSI.

Note that both of these gaps are finite and independent of the SNR. Therefore, we can conclude that the performances of both original and double-zipper ZigZag decoding algorithms approach that of the optimal time-sharing (which needs coordination, hence it is more difficult to implement) in the high SNR regime.

\section{NUMERICAL RESUlTS}

In this section, we compare the performance of ZigZag decoding with that of (coordinated) time-sharing in both perfect and imperfect CSI scenarios. We assume Rayleigh fading with unit variance channel gains, which are constant during the two time intervals. We have set the default values of the parameters as $N=600, \beta_{1}=0, \beta_{2}=0.025\left(L_{2}=15\right)$ and $\alpha=0.05$ (corresponding to $T=1230$ ). Also, an outage probability of $10 \%$ is considered.

The outage capacity with perfect CSI is plotted versus the SNR in Fig. 3 for different delay values $\left(\Delta L \triangleq L_{2}-L_{1}\right)$. It can be seen that the original ZigZag performs slightly better than the double-zipper ZigZag, owing to higher noise correlation. Furthermore, ZigZag decoding even outperforms the result in [21] for some SNR values (see (3)). Constant capacity gap between the ZigZag performance and the sum capacity at high SNRs is also clear, demonstrating the asymptotic optimality of this approach.

In order to have a more practical view, achievable rates of the time-sharing and the ZigZag decoding schemes with 


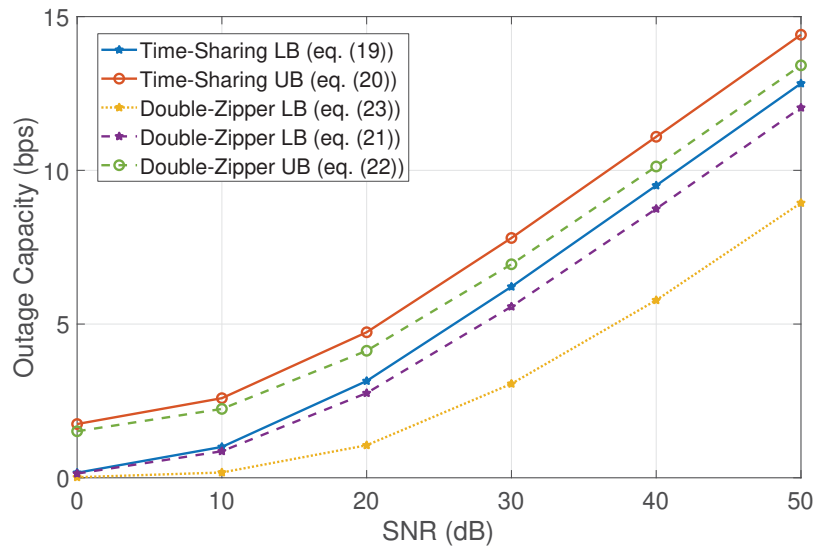

Fig. 5: Outage capacity with imperfect CSI.

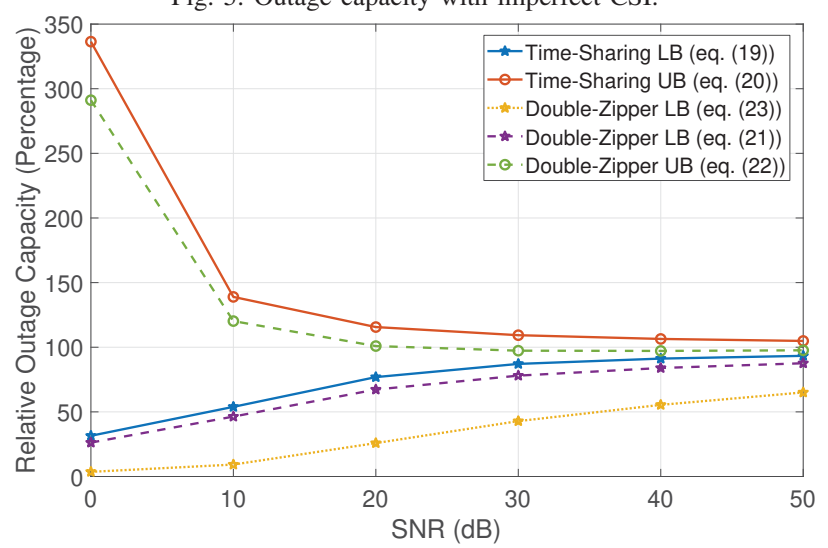

Fig. 6: Relative outage capacity with imperfect CSI.

finite alphabet signaling with quadrature amplitude modulation (QAM) are depicted in Fig. 4. As an example, for 16-QAM at an SNR of $40 \mathrm{~dB}$, at least $87 \%$ and $91 \%$ of the capacity of time-sharing can be achieved by the original and the doublezipper ZigZag algorithms, respectively. Clearly, the asymptotic performances of both ZigZag approaches are the same as the time-sharing scheme.

Next we consider the case of imperfect CSI with $c=1$ (i.e, $\sigma_{h}^{2}=\frac{1}{\rho}$ ). The outage and relative outage capacities are plotted versus SNR in Fig. 5 and Fig. 6, respectively. The relative capacities are normalized with respect to the timesharing sum-rate capacity with perfect CSI. It can be seen that the capacity gaps of both ZigZag algorithms fade away in high SNRs, even in the presence of imperfect CSI.

\section{CONCLUSIONS}

In this paper, we consider a two-user multiple access system with ZigZag decoding at the receiver. We first introduce a modified version of ZigZag, called double-zipper ZigZag, which decodes the received packets from both sides. By deriving some bounds on the achievable sum-rate of ZigZag decoding for non-unit gain channels with imperfect CSI, we show that the performance of ZigZag decoding approaches that of the optimal time-sharing in the high SNR regime. This is obtained without the need for any coordination, contrary to the time-sharing approach, and the performance is achieved by simple single user codes. Noting that there is no restriction on the codebooks, this approach can be a viable solution to the massive random access problem by extending the results to more than two users and by combining it with suitable medium access protocols.

\section{REFERENCES}

[1] R. Ahlswede, Multi-way communication channels," 2nd Int. Symp. Inf. Theory (ISIT), Tsahkadsor, Armenia, USSR, Sept. 2-8, 1971.

[2] H. Liao, Multiple access channels," Ph.D. dissertation, Dept. Electr. Eng., Univ. Hawaii, Honolulu, 1972.

[3] T. M. Cover and J. A. Thomas. Elements of Information Theory. New York: Wiley, 1991.

[4] R. G. Gallager, A perspective on multiaccess channels, IEEE Trans. Inf. Theory, vol. IT-31, pp. 124-142, Mar. 1985.

[5] R. Knopp, Coding and multiple-access over fading channels," Ph.D. dissertation, EPFL, Lausanne, Switzerland, 1997.

[6] N. Abramson, The ALOHA systemAnother alternative for computer communications," in Proc. AFIPS Conf., vol. 36, 1970, pp. 295298.

[7] E. Paolini, G. Liva and M. Chiani, "Coded slotted ALOHA: A graphbased method for uncoordinated multiple access," IEEE Trans. Inf. Theory, vol. 61, no. 12, pp. 6815-6832, Dec. 2015.

[8] S. Gollakota and D. Katabi, ZigZag decoding: Combating hidden terminals in wireless networks", in Proc. ACM SIGCOMM, New York, NY, USA, Aug. 2008, pp. 159170.

[9] Y. Polyanskiy, A perspective on massive random-access," 2017 IEEE Int. Symp. Inf. Theory (ISIT), Aachen, 2017, pp. 2523-2527.

[10] A. Vem, K. R. Narayanan, J. Cheng and J. Chamberland, A userindependent serial interference cancellation based coding scheme for the unsourced random access Gaussian channel," 2017 IEEE Information Theory Workshop (ITW), Kaohsiung, 2017, pp. 121-125.

[11] O. Ordentlich and Y. Polyanskiy, Low complexity schemes for the random access Gaussian channel," 2017 IEEE Int. Symp. Inf. Theory (ISIT), Aachen, 2017, pp. 2528-2532.

[12] J. Paek and M. J. Neely, Mathematical analysis of throughput bounds in random access with ZigZag decoding," 2009 7th Int. Symp. Modeling and Optimization in Mobile, Ad Hoc, and Wirel. Netw. (WiOpt), Seoul, 2009, pp. 1-7.

[13] A. ParandehGheibi, J. K. Sundararajan and M. Médard, Collision helps Algebraic collision recovery for wireless erasure networks," 2010 Third IEEE Int. Workshop Wirel. Netw. Coding (WiNC), Boston, MA, 2010, pp. 1-6.

[14] A. ParandehGheibi, J. K. Sundararajan and M. Médard, Acknowledgement design for collision-recovery-enabled wireless erasure networks,' 2010 48th Annu. Allerton Conf. Commun., Control, and Comput. (Allerton), Allerton, IL, 2010, pp. 435-442.

[15] A. S. Tehrani, A. G. Dimakis and M. J. Neely, SigSag: Iterative detection through soft message-passing," IEEE J. Sel. Topics Signal Process., vol. 5 , no. 8 , pp. $1512-1523$, Dec. 2011

[16] S. Ogata and K. Ishibashi, Application of ZigZag decoding in frameless ALOHA," IEEE Access, vol. 7, pp. 39528-39538, 2019.

[17] C. W. Sung and X. Gong, A ZigZag-decodable code with the MDS property for distributed storage systems, in Proc. 2013 IEEE Int. Symp. Inf. Theory (ISIT), 2013, pp. 341345.

[18] T. Nozaki, Fountain codes based on ZigZag decodable coding," 2014 Int. Symp. Inf. Theory and its Appl. (ISITA), Melbourne, VIC, 2014, pp. 274-278.

[19] A. Vem, K. R. Narayanan, J. Cheng and J. Chamberland, A userindependent serial interference cancellation based coding scheme for the unsourced random access Gaussian channel," 2017 IEEE Inf. Theory Workshop (ITW), Kaohsiung, 2017, pp. 121-125.

[20] A. El Gamal and Y. H. Kim, Network Information Theory. Cambridge: Cambridge University Press, 2011.

[21] A. S. Avestimehr, S. N. Diggavi and D. N. C. Tse, Wireless network information flow: A deterministic approach," IEEE Trans. Inf. Theory, vol. 57, no. 4, pp. 1872-1905, April 2011.

[22] M. Médard, The effect upon channel capacity in wireless communications of perfect and imperfect knowledge of the channel," IEEE Trans. Inf. Theory, vol. 46, no. 3, pp. 933-946, May 2000. 